\title{
ETUDE D'UN CHAMPIGNON PATHOGÈNE DU GENRE MONILIA
}

\author{
Par BROCQ-ROUSSEU, GUILLIERMOND et L. DES CILLEULS
}

Le Champignon qui fait l'objet de cette étude a été isolé sur un malade dont voici l'observation médicale résumée :

Le spahi K. Ahm., cl. 1903, entre à l'Infirmerie-Hôpital de l'Ecole de Cavalerie le 29 novembre 1924 pour bronchite généralisée, avec mauvais état général et $37^{\circ} 2$ de température. Il est dirigé quelques jours après sur les salles militaires de l'hospice mixte de Saumur avec le diagnostic de «pleurocongestion des deux bases pulmonaires, à forme apyrétique, avec prédominance ḋ gauche ». Ce cavalier, à son incorporation, ne présentait rien d'anormal. Ses poumons étaient mentionnés normaux. Son poids était de $66 \mathrm{kgr}$. pour $1-\mathrm{m} .77$ de taille, et son coefficient de robusticité était de 25 . Aucune trace d'albumine n'était constatée dans les urines. Aucun antécédent héréditaire n'était signalé.

En Algérie, il a fait un premier séjour à l'infirmerie pour bronchite. En France, il en fait un second du 28 octobre au 13 novembre 1924 pour bronchite légère. Pas de syphilis personnelle avouée.

Le 4 décembre 1924, jour de son admission à l'hospice de Saumur, sa température monte à $38^{\circ} 2$ dans la soirée, puis revient à la normale dès le lendemain, et s'y maintient les jours suivants jusqu'au 21 décembre. Pendant cette période l'état pulmonaire reste stationnaire.

Le 21 décembre, le malade accuse une gêne respiratoire assez accentuée, son pouls est rapide, et sa température atteint $40^{\circ} 9$. On note au poumon droit les signes cliniques d'une pneumonie.

Le lendemain, une injection intraveineuse de $20 \mathrm{cc}$. de sérum antipneumococcique est pratiquée ; elle est renouvelée le 27 décembre.

Dix jours après, la température tombe à $37^{\circ} 5$ le matin et à $37^{\circ} 8$ le soir: la phase aiguë semble s'apaiser. Mais, alors que la convalescence normale avec apyrexie pouvait être escomptée, la fièvre s'installe aux environs de $38^{\circ}$ et s'y maintient désormais.

Les signes d'auscultation, l'accentuation du mauvais état général, l'amaigrissement, font.penser à un syndrome de tuberculose cavitaire de la base pulmonaire droite. Il faut noter également que l'expectoration est très abondante et à caractère nummulaire, mais sans odeur gangréneuse. Le malade remplit, en effet, un peu plus de deux crachoirs par 24 heures. Deux examens bactériologiques des crachats sont effectués, à cette époque, mais ne décèlent pas la présence de bacilles de Koch.

Au début de février, l'état toujours précaire du malade permet toutefois un examen radioscopique pulmonaire. Il est effectué le 4 février par le Docteur Moreau, radiologiste de l'hospice mixte, qui note : «En

Andales de Parasitologie, T. V, $x^{\circ} 1 .-1^{\text {er }}$ janvier 1927, p. 48-62. 
avant et à droite, voile du.sommet. Côté gauche normal. Grosse réaction hilaire et péribronchique. Médiastin s'éclairant mal. En arrièré, voile étendu de la base-droite. Cul-de-sac costo-diaphragmatique ne s'ouvrant pas. Côté gauche normal. »

L'examen des urines donne les résultats suivants : « Aspect trouble ; réaction franchement alcaline; densité 1.026 ; dépôt abondant soluble dans l'acide acétique (phosphates). Présence d'albumine à la dose de 0 gr. 20 par litre. „ Le 11 février, une nouvelle recherche du bacille de Koch dans les crachats homogénéisés reste négative.

Au cours des semaines qui suivent, aucune amélioration n'est constatée. Les signes pulmonaires sont sensiblement les mêmes, mais le mauvais état général s'accentue. L'expectoration demeure très abondante, sans odeur fétide, et fatigue beaucoup le malade par sa fréquence.

Le 17 mars, un quatrième examen bactériologique des crachats reste négatif, quant à la présence du bacille de Koch. On inocule deux cobayes le lendemain, et ceux-ci meurent le 28 mars. Leur autopsie fournit les résultats suivants :

« Congestion intestinale avec exsudat péritonéal sérohémorragique. Abcès au point d'inoculation avec zone mortifiée. Le frottis de cette dernière ne décèle pas de bacilles de Koch. L'examen du sang ne montre pas d'éléments pathogènes. » Le 2 et 6 avril, la recherche du bacille de Koch en est encore négative.

En présence de l'état du malade, de l'absence du bacille de Koch dans son expectoration extrêmement abondante ef sans odeur fétide, et après avoir éliminé l'hypothèse d'une dilatation bronchique ou d'une gangrène pulmonaire, on envisage la possibiiité d'une mycose du poumon, et des recherches microscopiques sont orientées de ce côté. Elles font constater sur les préparations faites des filaments mycéliens abondants.

On prescrit au malade des gargarismes et des lavages de bouche à l'eau oxygénée diluée au 1/4, afin d'éviter que les moisissures et les germes banaux saprophytes ne masquent l'agent pathogène. Les examens microscopiques sont effectués sur crachat frais, recueilli dès l'expectoration, et après gargarisme. On pratique, en outre, des ensemencements sur milieu de Sabouraud.

Les recherches sont positives, et l'on institue dès le 23 avril un traitement ioduré ( $3 \mathrm{gr}$. d'iodure de potassium pro die).

Il faut noter que le poids du malade, consigné le 21 mars, atteignait $56 \mathrm{kgr}$. 400 , soit un amaigrissement de près de $10 \mathrm{kgr}$. depuis l'incorporation.

La dose d'iodure de potassium est élevée progressivement jusqu'à 8 gr.

Sous l'influence du traitement, l'état général s'amende rapidement. L'expectoration diminue peúa peu, et le malade qui ne dormait plus, en raison de sa toux incessante, retrouve un sommeil réparateur. Les éléments mycéliens ne se rencontrent plus dans les crachats. Le 13 mai, le malade ne crache plus, et les signes pulmonaires ont presque totalement disparu. On cesse alors l'administration de l'iodure de potassium pris pendant 20 jours. 
Le 16 mai, on pèse à nouveau le malade: son poids est de $67 \mathrm{kgr}$, c'est-à-dire qu'il présente une augmentation de $11 \mathrm{kgr}$. en deux mois et demi.

Le 4 juin, lé malade sort entièrement guéri de l'hôpital, après y avoir séjourné plus de 6 mois, et reprend immédiatement son service qu'il effectue normalement jusqu'au 25 aoùt 1925 , date à laquelle il est rapatrié en Algérie, pour sa libération de service.

En résumé, l'histoire clinique de cet homme est la suivante : admis dans un service hospitalier pour bronchite en fin d'octobre 1924 , il a présenté, quelques jours après son admission, des signes de pleuro-congestion pulmonaire bilatérale apyrétique, compliqués ensuite d'une poussée pneumonique droite, donnant ultérieurement à penser, par les signes cliniques observés, à l'évolution d'une tuberculose cavitaire, avec déchéancé organique marquée.

L'absence de bacille de Koch dans l'expectoration extrèmement abondante, et non fétide de ce malade, traité depuis près de 5 mois dans un service hospitalier sans aucune amélioration, a orienté les recherches microscopiques vers la possibilité d'une mycose pulmonaire, qui a été reconnue existante, et qui a cédé très rapidement au traitement ioduré prescrit pendant 20 jours. Sous l'influence de ce dernier, le malade a repris 11 kilogr. et est sorti guéri de l'Hòpital.

\section{Caractères des cultures}

Moût de bière liquide. - Le champignon végète abondamment dans ce milieu, entre 25 et $40^{\circ}$; il se développe d'abord au fond du tube sous forme d'un dépôt blanchâtre; puis il montre une tendance à monter le long des parois du tube où il forme un revêtement blanchâtre, puis, il produit un anneau à la surface. En même temps, on constate souvent la présence sur les bords du tube, à la surface du liquide, de petits grumeaux-de levures en suspension, et le liquide donne les indices très nets d'une fermentation. A partir de ce moment, l'anneau se développe de plus en plus, et, vers le quinzième jour, il forme-un large revêtement tout autour de la paroi, au-dessus de la surface du liquide; il prend une couleur jaune-brunâtre. Nous n'avons pas observé la formation d'un voile, même au bout de deux mois.

Eau de levure. - Au bout de deux jours, apparition d'îlots grisâtres, qui se réunissent bientôt pour former un voile complet, très délicat.

Moủt gélosé, eau de levure gélosée, gélose de Gorodkowa. - Sur 
ces milieux, le champignon produit un enduit blanchâtre, d'aspect humide, tout à fait semblable à celui d'une levure.

Carolte, pomme de terre. - Colonies blanches qui confluent au bout de peu de temps.

Gélose. - Culture bien développée le long de la strie d'ensemencement ; trainée blanche presque transparente, épaisse, en relief. Vers le sommet, colonies punctiformes plus blanches.

Gélose Sabouraud. - Mêmes caractères ; culture plus blanche et plus abondante, étalée en un large placard, avec, sur les bords, des colonies punctiformes, non encore confluentes.

Gélose au rouge neutre. - Développement très faible en piqûre, en profondeur ; culture seulement à la surface, au point d'inoculation; pas de virage.

Gélose au sous-acétate de plomb. - En piqûre, pousse en surface et dans la profondeur ; pas de noircissement.

Gélose tournesolée saccharosée. - Colonie blanchâtre, le long de la strie d'ensemencement, s'étendant plus tard à toute la surface. Au début, rougissement léger du milieu qui redevient bleu au bout de très peu de temps.

Gélose tournesolée lactosée. - Développement très faible ; pas de changement de coloration du milieu.

Gélatine. - En surface, colonie crémeuse, blanche, le long de la strie; développement peu abondant. En piqûre, culture le long de la piqûre, en forme d'infundibulum, et cultures perpendiculaires, donnant l'impression d'une brosse à pipe.

Sérum coagulé. - Culture peu abondante, formant un dépoli discret. Très fines colonies, avec bavures blanc crémeux.

Bouillon Martin. - Le milieu se trouble uniformément et est légèrement grumeleux. Dépôt de grumeaux de taille variable, s'élevant par agitation.

Bouillon sérum. - Zònes nuageuses, grumeleuses au milieu du tube. Dépôt abondant. Culture moins forte qu'en bouillon Martin.

Eau peptonée $(2,50 / 0)$. - Culture faible, dépôt au fond du tube. Plus tard, la culture devient abondante. Pas de formation d'indol.

Lait. - Culture et coagulation du lait en 12 à 15 jours; puis, digestion ultérieure du caillot. Si l'on ajoute du tournesol au milieu, il n'y a pas de changement de coloration. La coagulation n'est done, pas due à une augmentation de l'acidité du milieu ; il y a, par conséquent, secrétion de présure, coagulant le lait; puis secrétion d'une caséase digérant le caillot. 
Albumine (au tiers). - Dans cette solution, culture, sous forme de grumeaux. On peut mettre en évidence la formation de trypsine, par la digestion de cette albumine qui a donné des produits de désintégration que l'on caractérise dans la solution, par le réactif de Millon, et le biuret.

Amidon (à $100 / 0$ ). - Culture, sans formation de sucre.

CARACTÉRES MORPHOLOGIQUES ; FORMES ET DIMENSIONS DES CELLULES

Sur moût de bière, entre 25 et $40^{\circ}$, après 24 heures, le champignon se présente d'abord presque exclusivement sous forme de levures rondes ou ovoïdes, parfois réunies par petits groupes (Fig. 1, 1), mais bientôt, on voit apparaître, à côté de ces levures, quelques filaments mycéliens, résultant de la croissance de cellules levures qui s'allongent en une sorte de tube germinatif, lequel, ensuite, se cloisonne et se ramifie, formant un véritable mycélium. Au bout de 15 jours à trois semaines, la végétation se compose d'un mélange de levures et de filaments mycéliens (fig. 1, 2). Les levures ont des formes variables; à còté de cellules sphériques ou ovoïdes, on en trouve d'allongées, de cylindriques ou en forme de saucisse, presque toutes renferment un gros globule graisseux. Les filaments offrent tous les degrés de développement. Certains sont très courts, formés par trois, quatre cellules non ramifiées, d'autres, très allongés et ramifiés, constituent un mycélium typique. Les cellules de ce mycélium forment fréquemment, 'par bourgeonnement latéral ou terminal de nouvelles levures. Ce mycélium est tantòt formé par des filaments très mincés et d'un calibre égal, tantôt par des filaments renflés en certains endroits. Bien que les filaments soient assez nombreux et parfois très développés, les levures prédominent toujours, et il est parfois nécessaire de rechercher les filaments avec attention pour les observer, surtout dans les vieilles cultures dans lesquelles les articles du mycélium ont une tendance à se dissocier. On rencontre enfin, très fréquemment, des cellułes levures géantes ; celles-ci sont le plus souvent rondes, et offrent parfisis une paroi épaissie ; elles donnent naissance, sur leur pourtour, à des levures, en vidant leur contenu qui se réduit finalement à quelques granulations graisseuses, et peuvent déchirer leur membrane. Ce sont certainement des formes d'involution, en voie de dégénérescence. On observe le même aspect, après 2 mois. La dimension des cellules varie de $2,8 \mu$ à $8 \mu$ de diamètre, pour les cellules rondes; les cellules ovoïdes ou allongées mesurent de 4 à $8,5 \mu$ de largeur sur 6 à $10 \mu$ de longueur. Les cellules géantes ont de 10,5 à $18 \mu$ de diamètre, et peuvent atteindre jusqu'à 30 et $40 \mu$. 


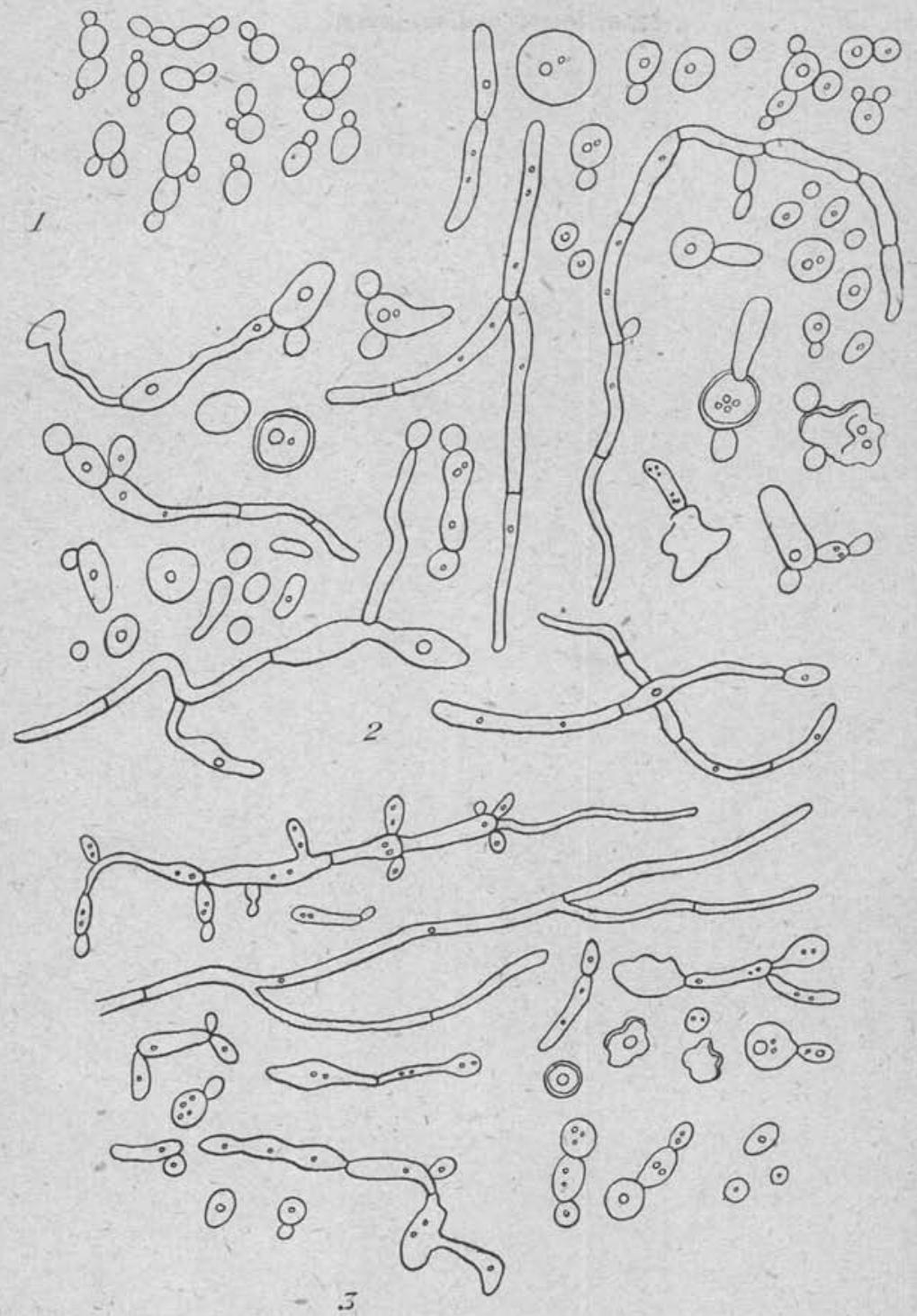

Fig, 1. - Culture sur moût de bière liquide. 1 , Agée de 24 heures ; 2 , de 15 jours; 3 , anneau au bout de 2 mois.

Sur moùt de bière gélosé, le Champignon se présente au bout de 24 heures, sous forme de levures rondes et surtout ovoïdes, rarement allongées, parfois réunies par petits groupes (fig. 2, 1), puis 
bientôt dès le deuxième jour, apparaissent des formations mycéliennes (fig. 2,2 ). Au bout de 15 jours à trois semaines, il présente un mélange de filaments à tous les degrés de développement dont les uns se ramifient abondamment, sous forme de rameaux souvent sinueux, et de levures présentant les formes les plus diverses : rondes, ovoïdes, cylindriques, très allongées, et qui, presque toutes renferment un ou plusieurs gros globules graisseux à leur inté-

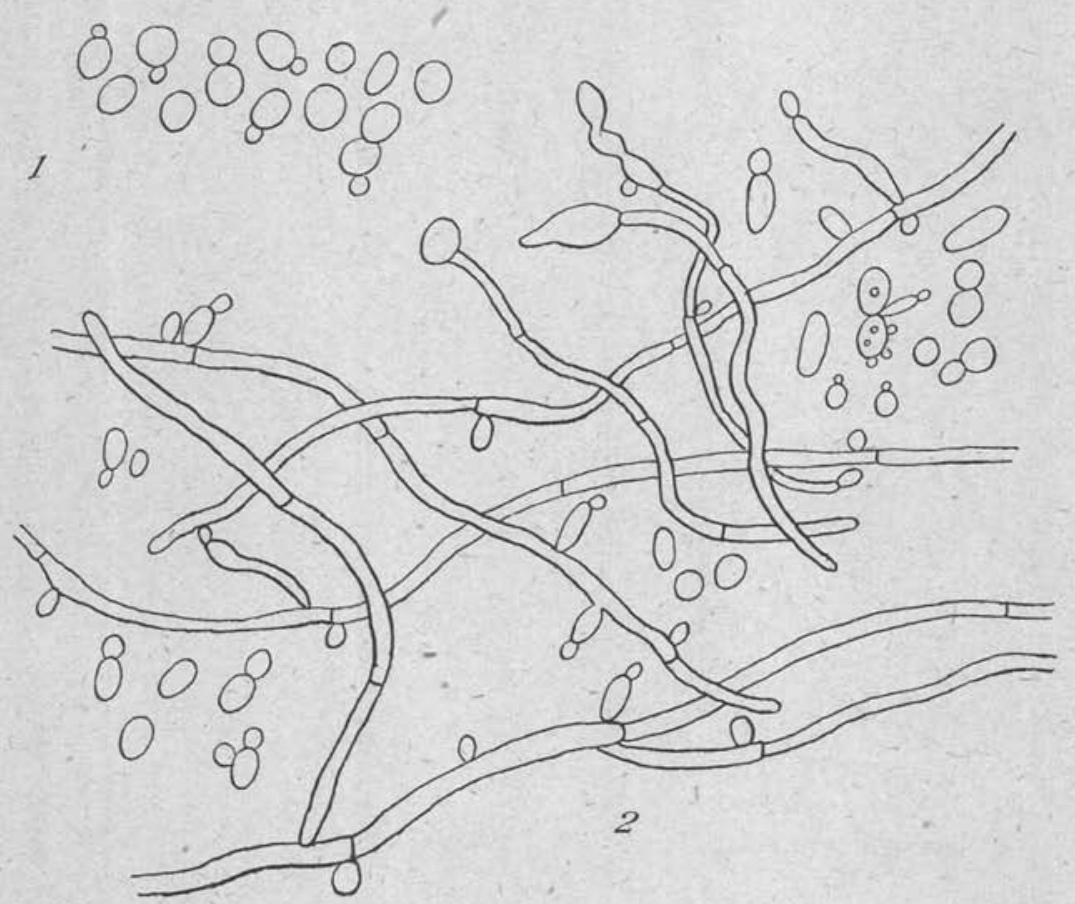

Fig. 2. - Culture sur moût de bière gêlosé. 1 , Agée de 1 jour ; 2 , de 48 heures.

rieur. On remarque, en oủtre, de nombreuses cellules géantes semblables à celles observées sur le moût liquide. Ces cellules bourgeonnent sur leur pourtour et produisent, tantôt des levures, tantôt des filaments mycéliens. Leur membrane est souvent épaissie et irrégulière, et leur contenu se réduit à quelques granulations graisseuses (fig. 3 ).

Sur carotte, on observe d'abord des levures rondes, et plus souvent ovoïdes (fig. 4,1 ), puis au bout de 48 heures apparaissent des formations mycéliennes (fig. 4, 2). Après quinze jours, la culture présente un mélange de levures rondes, ovoïdes ou allongées, par- 
fois en forme de boudin, avec un gros globule graisseux àu centre, et de filaments mycéliens plus ou moins développés. La végétation sur gélose de Gorodkowa est composée, au bout de 24 heures, exclusivement par des levures rondes ou ovoïdes isolées ou réunies par petits groupes (fig. 5,1 ). Au bout de 8 jours, on trouve un mélange



Fí. 3. - Culture sur moût de bière gélosê âgée de 15 jours.

de levures, renfermant à leur intérieur un gros globule graisseux, et de filaments mycéliens donnant naissance à des levures, par bourgeonnement (fig. 5, 2). Après 15 jours, les levures sont toujours nombreuses et offrent des formes très variables, rondes, ovoïdes, allongées. Parmi elles, se trouvent de nombreuses cellules géantes qui se vident après avoir donné naissance sur leur pourtour à des levures ou à des filaments mycéliens. On trouve un très grand nombre de filaments allongés, très ramifiés qui par bourgeonnement 
latéral donnent de nombreuses levures (fig. 5, 3). Dans aucun autre milieu, le mycélium n'atteint un aussi grand développement.

Dans le voile développé sur eau de levure, le Champignon se présente au bout de 48 heures sous forme de levures rondes ou ovoïdes, le plus souvent réunies par petits groupes (fig. 4,3 ). Au bout de 3 jours, les cellules les plus grandes et les plus anciennes de cha-

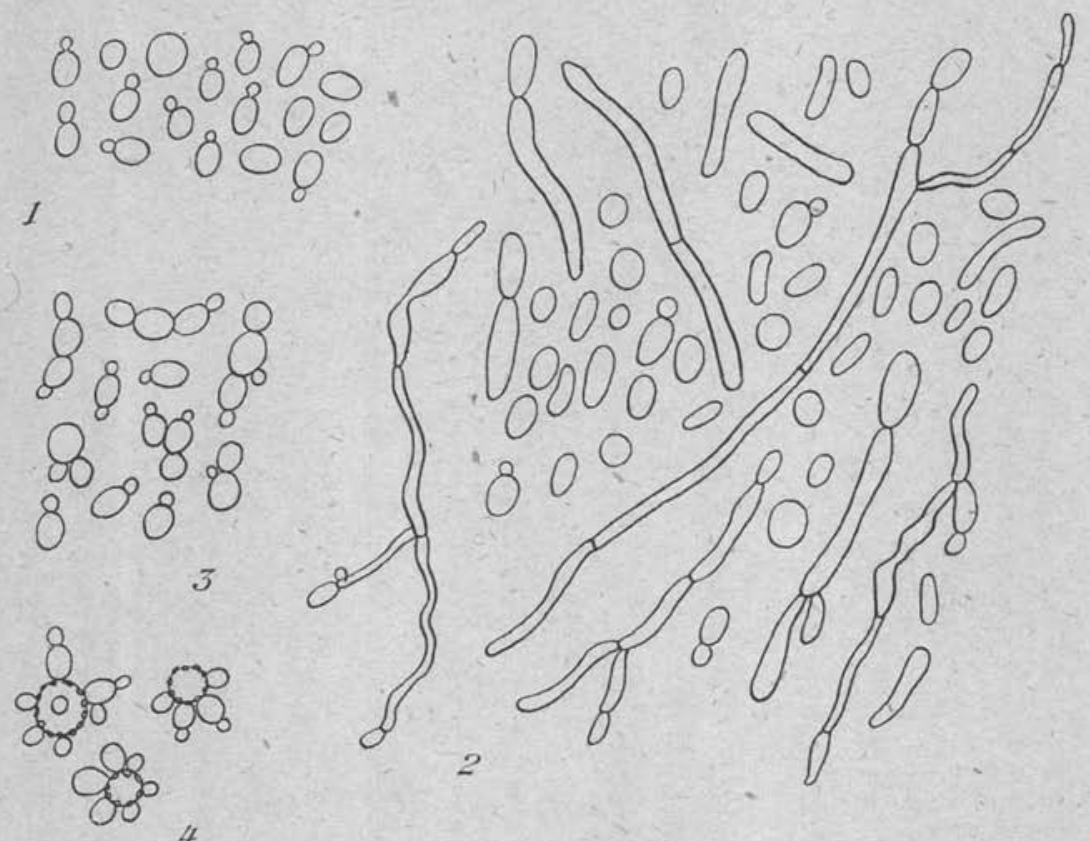

4

Fıg. 4. - 1, Culture sur carotte âgée de 24 heures ; 2 , culture sur carotte âgée de 48 heures; 3 , voile sur eau de levures au bout de 48 heures; 4 , voile sur eau de levures au bout de 8 jours.

levures issues de leur bourgeonnement, et qui en occupent le centre, forment sur leurs parois de petites protubérances verruqueuses (fig. 4, 4), qui lui donnent un peu l'aspect des cellules durables, notamment de celles qui ont été décrites par Vuillemin et Legrain dans le Saccharomyces granulomatogenes (1). Mais, en observant les cellules, on constate qu'elles ont un contenu dense et contracté au milieu de la cellule, qui dégénère peu à peu. Ces cellules ne tardent pas à se vider et finissent par ne plus renfermer que des granulacune des colonies formées par la réunion d'un certain nombre de

(1) Sur un cas de saccharomycose humaine. Arch. de Parasilologie, 1900. 


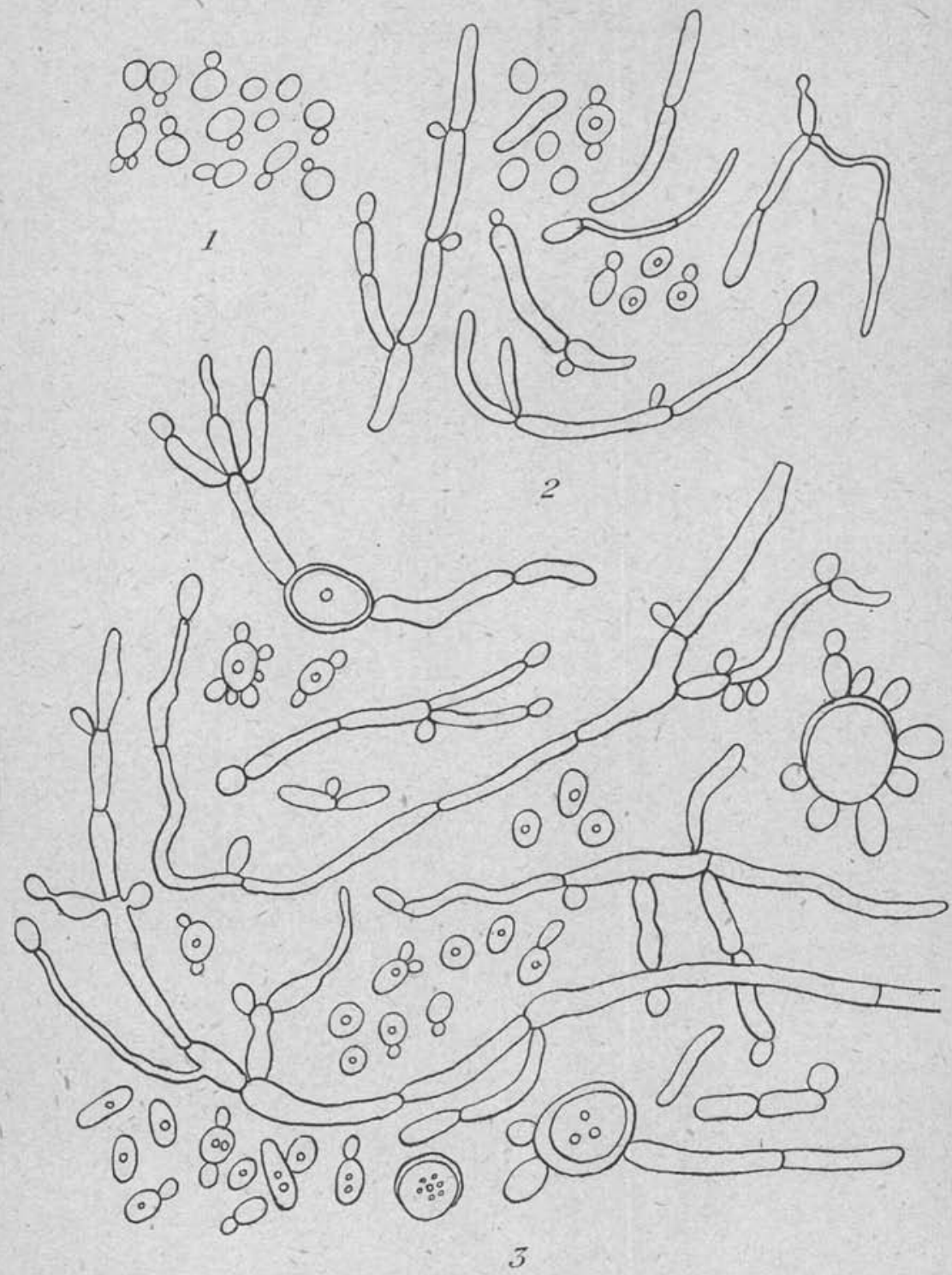

Fig. 5. - Culture sur gélose de Gorodkowa. 1, Au bout d'un jour ; 2 , au bout de 8 jours ; 3 , au bout de 3 semaines.

tions graisseuses, tandis que leur membrane prend un contour irrégulier. Si l'on colore vitalement la levure par une solution très diluée de bleu de méthylène, on constate que ces cellules se colorent 
immédiatement quand elles ne sont pas encore vides, et que leur contenu contracté prend une teinte homogène ; les autres cellules au contraire se colorent beaucoup plus lentement, et le bleu de méthylène se fixe chez elles exclusivement dans la vacuole. Ces cellules ne sont donc pas des cellules durables et l'on doit les considérer comme des cellules géantes, formes de dégénérescence.

\section{Sporulation}

Aucune sporulation n'a été constatée dans les conditions où les levures forment des asques : gélose de Gorodkowa, blocs de plâtre, tranches de carotte.

\section{Colonies gÉantes}

Au bout de 15 jours, la colonie géante sur moût de bière gélosé ofire les dimensions d'une pièce de einq franes. Elle est ronde, de couleur blanc jaunâtre et d'aspect humide. Son centre montre une structure granulo-réticulée; la périphérie est lisse et parcourue simplement par quelques sillons rayonnant à partir du centre, et se terminant dans une échancrure du bord, déterminant de larges lobes. Le bord est uni.

$\mathrm{Au}$ bout de 2 mois, la colonie s'est beaucoup accrue : elle conserve au centre une partie plus épaisse et d'aspect mésentéroïde, entouré d’une large zóne lisse parcourue par quelques sillons rayonnant autour du centre et se terminant dans une échancrure du bord. Mais le bord se prolonge en outre par une zone moins épaisse et très finement festonnée.

Sur gélatine au moût de bière, en tube à essais, la liquéfaction commence au bout de 10 jours : à ce moment, toute la partie supérieure du substratum est liquéfiée. La liquéfaction se continue peu à peu jusqu'au fond du substratum ; au bout de 2 mois, elle est complète et le champignon forme une sorte de voile à la surface du liquide.

\section{Coloration}

Le champignon se colore par toutes les méthodes uśuelles. Il n'est ni acido- ni alcoolo-résistant.

\section{Caractères BIOLOGIQues}

Le champignon pousse à l'air et en anaérobie.

Températures de croissance. - Sur moût de bière, le champignon ne paraît pas se développer au-dessous de $10^{\circ}$, et, à cette tempéra- 
ture, il ne végète que lentement. Sa croissance est très rapide entre $25^{\circ}$ et $40^{\circ}$. Elle est à peine plus lente à $40^{\circ}$ qu'à $35^{\circ}$. L'optimum de croissance sur moût de bière est situé entre $30^{\circ}$ et $40^{\circ}$.

A $45^{\circ}$, la croissance se ralentit et cesse entre $46^{\circ}$ et $47^{\circ}$. A partir de $40^{\circ}$, le champignon ne forme plus d'anneau sur moût de bière et ne végète que sous forme de dépôt.

Résistance à la chaleur. - Cette résistancê a été essayée avec des cultures de 48 heures, mises en ampoules de 1 cc., plongées dans un bain d'huile chauffé à différentes températures.

Les températures ont été essayées de $55^{\circ}$ à $100^{\circ}$, et l'action a été contrôlée par deux cultures successives, l'une faite de suite avec l'ampoule sortant du bain-marie, l'autre 15 jours après.

Le résultat a été le suivant :

$55^{\circ}$ pendant 5 minutes, développement.

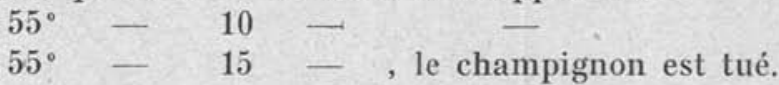

Toutes les cultureś essayées à des températures supérieures à $55^{\circ}$, quel que soit le temps, ont été tuées. Nous avons donc la limite inférieure de sa résistance à la chaleur : $55^{\circ}$ pendant 15 minutes.

Action sur les sucres. - Le champignon invertit le saccharose. Par la méthode des petites fermentations de Lindner, il produit une fermentation assez active du maltose, et une fermentation moyenne du dextrose et du lévulose. Il fait faiblement fermenter le mannose et aussi, semble-t-il, la dextrine et n'a aucune action sur le galactose, le lactose et le raffinose.

Diastases. - Nous avons dit plus haut que le champignon secrète une présure, une caséase et une trypsine. Il ne secrète pas d'amylase.

Résistance à l'iodure de potassium. - L'affection causée par ce champignon, lorsque le diagnostic fut posé, céda à l'administration d'iodure de potassium. Le fait est bien établi pour toutes les mycoses, et ce traitement est classique.

L'un de nous avait essayé autrefois avec un autre champignon pathogène, Acremonium Potronii Vuill., sa résistance à l'iodure, et était arrivé à le cultiver dans des bouillons contenant $300 / 0$ d'iodure.

Nous avons recherché cette résistance pour ce nouveau champignon. Il fut entraîné à vivre dans des bouillons auxquels on ajoutait des gouttes d'une solution d'iodure à $200 / 0$, puis avec une solution à $1000 / 0$, puis enfin par l'adjonction de doses, en poids, d'iodure au bouillon. Nous sommes arrivés à le cultiver dans des 
milieux contenant $300 / 0$ d'iodure. A $350 / 0$, il ne pousse plus et il est tué.

Nous ne pouvons nous èmpècher de remarquer que la dose est la même $(300 / 0)$ pour deux champignons très différents, et dans des expériences faites à 15 années de distance.

L'intérêt de la question réside dans le fait que, si des champignons sont capables de s'habituer en cultures, à la présence d'iodure de potassium, il peut en être de même dans l'organisme, et que, alors, les petites doses thérapeutiques d'iodure devraient ètre proscrites, pour n'employer que des doses aussi massives que possible, dès le début du traitement (1).

Toxine. - Nous avons cherché si le champignon sécrétait une toxine. Une culture de 12 jours en bouillon est filtrée sur bougie L3. et le filtrat est injecté à un lapin, dans l'oreille, et à deux cobayes, l'un sous la peau, l'autre dans le péritoine.

Aucun des animaux n'a présenté de troubles consécutifs. Le champignon ne sécrète donc aucun produit toxique.

Fixation du complément. - Nous avons recherché si l'injection du champignon aux animaux faisait apparaitre une sensibilisatrice dans leur sérum, et si elle était spécifique. Nous avons pris comme antigènes le champignon lui-même, une levure de champagne et un cryptocoque. Les animaux injectés ont été sacrifiés 8 jours après l'inoculation, par saignée à blanc.

1) Cobaye injecté dans le péritoine :

Champignoń, fixe 1 dose d'alexine.

Levure de champagne, réaction négative.

Le sérum d'un cobaye témoin donne une réaction négative avec le champignon.

2) Cobaye inoculé sous la peau :

Champignon, réaction négative.

Levure de champagne, réaction négative.

3) Lapin inoculé dans la veine :

Les trois antigènes, champignon, levure et cryptocoque, fixent tous 3 doses d'alexine.

Il est done impossible de conclure positivement à la formation d'anticorps dans le sérum des animaux inoculés, et lorsqu'il y a une sensibilisatrice, il n'y a aucune spécificité.

(1) Gais (E.) et Broce-Rousseu. - Résistance à l'iodure de potassium de l'Acremonium potronii. Soc. de Biol., 16 déc. 1912, p. 46.

Broco-Rousseu. - Etude de l'Acremonium potronii. Revue gén. de Botanique, XXVI, 1914, p. 150 . 
Pathogénie. - Le champignon est pathogène pour le lapin et le cobaye ; le rat blanc parait être réfractaire.

Voici quelques indications concernant cette question :

Lapin 1, poids 1.760 grammes. Reçoit dans la jugulaire, le 24-7-25, 1 cc. de culture de 24 heures en bouillon.

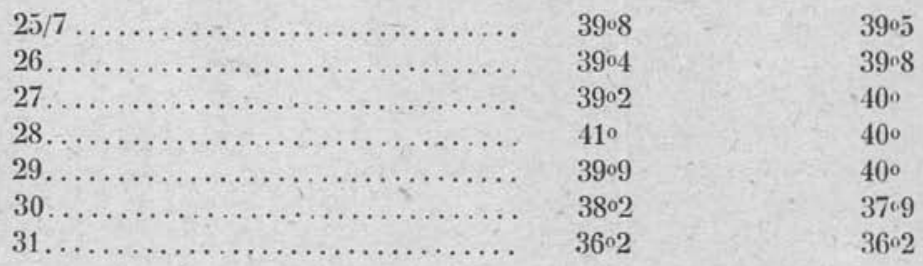

Mort le 31 , en hypothermie ; poids 1.120 ; il a done perdu 640 grammes en 7 jours. On trouve à l'autopsie de la congestion de l'intestin grêle, du mésentère et du foie.

Les examens par frottis du sang du cœur et de la rate ne donnent rien ; ceux du rein montrent quelques formes levures.

Les ensemencements du sang du cœur et de la moelle osseuse donnent des cultures pures en 12 heures; on y trouve des formes levures et des formes mycéliennes.

Lapin 2, inoculé le 11-8-25 avec $1 \mathrm{cc}$. de culture de 24 heures en bouillon, dans la veine.

Mort le 20-9. Emaciation extrême, rétention urinaire, dégénérescence rénale. L'examen du sang, de la rate, du rein, des poumons, du foie, montre de petites formes levures.

Le sang du cæur, en bouillon et sur gélose, donne une culture de formes levures.

Lapin 3, inoculé le 20-1-26 avec 1 ec., in veine, de culture de 24 heures en bouillon Martin. Poids 1.670 gr.

Mort le $26-1$; poids 1.240 ; perte 430 grammes en 4 jours.

Foie très congestionné ainsi que les vaisseaux mésentériques.

L'ensemencement de la bile et du rein donne une culture abondante ; le sang et le liquide céphalo-rachidien ne donnent rien.

L'examen direct du foie, du rein, de la bile, du sang du cœur montre des formes levures.

Lapin 4, inoculé le 11-2-26 avec $1 \mathrm{cc}$. dans la veine. Saigné à blanc le 18-2-26.

Lésions généralisées du rein sous forme de petits points blancs ; petit abcès du foie.

L'ensemencement du rein donne une culture abondante. 
Cobaye 1, inoculé le 6-8-25 avec 1 cc., in veine, de culture de 24 heures ; poids 550 gr. ; mort le $14-8$; poids 520 gr.

Pleuro-péricardite hémorragique; péricarde gonflé de sang; îlots de congestion dans le poumon; épanchement péritonéal hémorragique; foie volumineux, friable, dégénérescence blanchâtre par larges îlots ; rate décolorée, friable, reins gonflés, mous.

Cobaye 2, 1 ce. sous la peau le 11-2-26 ; saigné le 18-2.

Abcès caséeux au point d'inoculation, donnant une culture de tevure.

Rat blanc. Les inoculations dans le péritoine et le poumon n'ont rien donné.

\section{Afrinités -}

Par sa végétation à la fois sous forme de levures et de filaments mycéliens très développés, ainsi que par son absence de sporulation, le champignon doit être rapporté au genre Monilia Bonorden.

De nombreuses Monilia ont été isolées des crachats provenant de malades atteints de bronchomycoses ; elles ont été réunies par Castellani dans le groupe du Monilia tropicalis. On a isolé aussi dans les mycoses pulmonaires $M$. pulmonalis Mautner; M. brasiliensis de Magalhaès.

La description de toutes ces espèces est insuffisante pour nous permettre de rattacher à l'une d'elles l'espèce que nous venons de décrire. Cependant, certains caractères ne permettent de l'assimiler à aucune d'ellés et il semble qu'elle puisse être considérée comme une espèce nouvelle (1).

(1) Aucune de ces espèces ne présente en effet les caractères de notre Monilia. La couleur blanc jaunâtre de sa colonie géante l'éloigne de Monilia guilliermondi (colonie géante jaunâtre ou brunâtre), de $M$. rosea (colonie géante rose) et de $M$. zeylanica (colonie géante jaunâtre ou brunâtre). L'absence de voile en milieu liquide ne permet pas de la rapporter aux Monilia alba, albicans, balcanica, guilliermondi, nitida, parakrusei, paratropicalis et pulmonatis, qui forment au contraire un voile. Son action vis-à-vis des sucres l'écarte de $M$. alba, albicans, chalmersi, enterica, facalis, insolita, macedoniensis, metalondinensis, metatropicalis, negrici, nivea, parachalmersi, paratropicalis, pseudolondinensis, pseudolondinoïdes, pseudometalondinensis, psendotropicalis, pulmonalis, thoi, tropicalis, qui font fermenter le galactose, de $M$. pseudotropicalis, qui fait fermenter le lactose, de M. insolita, negrici, nivea, guilliermondi et leytonica, qui donnent une fermentation du raffinose et de $M$. balcanica, krusei, parabalcanica, parachalmersi, parakrusei, psendognilliermondi et tropicalis, qui n'agissent pas sur te maltose. Par sa propriété de liquifier la gêlatine, elle se rapproche de M. albicans et parachalmersi et par son action sur la dextrine de M. pseudolondinensis et pseudolondinoïdes, mais d'autres caractères l'éloignent de ces espèces. (Castellani et Chalmers. - Manual of tropical Medicin, 1919, p. 1082-1083). 